\title{
Experience in Using Liquid Cytology in the Diagnosis of Endometrial Pathologies
}

\author{
Kramarskiy Vladimir* \\ Irkutsk State Medical University, Russia
}

*Corresponding author: Kramarskiy Vladimir, Department of Obstetrics and Gynecology, Irkutsk State Medical University, Russia.
Received Date: May 10, 2020

Published Date: May 20, 2020

\section{Introduction}

The method of liquid Cytology is the most promising in the screening diagnosis of precancerous diseases of the cervix [1]. At the same time, various types of endometrial pathology cannot always be verified based on the results of liquid Cytology, despite its high prognostic value in the diagnosis of endometrial cancer.

\section{Purpose of the Study}

The purpose of our study was to compare the histological results of endometrial pathology and conclusions of liquid Cytology diagnostics.

\section{Material and Methods}

Sampling of material from the uterine cavity for liquid Cytology was performed according to the standard method in the second phase of the menstrual cycle with the placement of the obtained material in a specific liquid. Diagnostic scraping of the walls of the uterine cavity was performed after taking the material for liquid Cytology [2].

A total of 88 women were examined with suspected endometrial hyperplasia, glandular fibrotic polyp, adenomyosis, chronic endometritis, endometrial adenocarcinoma, and uterine fibroids.

Women with vaginal manipulations, hormone therapy, and recent (within 2 days) sexual contact were excluded from the study, signs of endometritis that use COCA for contraception.

\section{Results}

The Average age of the women surveyed was $36.8 \pm 1.7$ years. All the women had a history of childbirth. Medical abortions in 52 women. Complications after a medical abortion in 18. Uterine fibroids in 20 women.
The coincidence of the cytological conclusion and histological examination was noted in 47 (53.4\%) observations. In 41(46.6\%) cases, the findings of histological examination and liquid Cytology did not match. Out of 7 cases histologically diagnosed with endometrial adenocarcinoma, the result of liquid Cytology coincided with the histological conclusion only in 3 (42.9\%) cases. In the presence of endometrial hyperplasia (37 cases), according to histological research, the identical pathology was confirmed by liquid Cytology only in 12 (32.4\%) cases [3]. Atrophic endometrium was detected in 39 women based on histological examination. Cytological confirmation of the histological result of the study in these women was confirmed in 22 (56.4\%) cases.

Thus, even when the visible benefits of liquid-based Cytology of abnormalities of the endometrium ( the speed of obtaining results , quality material, multiple studies without re-sampling, and HPV PCR study) frequency of coincidence of the histological result and outcome of liquid based Cytology at different pathological States of the endometrium according to our data, ranged from $32.4 \%$ to $56.4 \%$.

\section{Conclusion}

The method of liquid Cytology, with all its advantages, does not have an absolute degree of accuracy in the diagnosis of pathological conditions of the endometrium.

Liquid Cytology for screening endometrial pathology should be accompanied by additional histological examination.

\section{Acknowledgement}

None. 


\section{Conflict of Interest}

No conflict of interest.

\section{References}

1. Chepurnaya Y (2005) Clinical significance of liquid Cytology in the diagnosis of cervical diseases.

2. Protasova AE. Yu Raskin GA, Orlova RV (2011) Using the method of liquid Cytology and immunochemical endometrial research in patients with breast cancer while taking tamoxifen. Questions of Oncology : 675-679.
3. Yang X, Ma K, Chen R, Zhao I, Wu C, et al. (2017) Liquid-based endometrial cytology associated with curettage in the investigation of endometrial carcinoma in a population of 1987 women. Gynecologic Oncology 296: 95-105. 